\title{
YOUR COLOUR MEMORY Y LA PELL DE LA PELL: DEL ARTISTA AL CIENTÍFICO
}

\author{
Cristina Pastó Aguilà \\ Eugènia Agustí Camí
}

Universidad de Barcelona, Dpto. de Pintura

\section{Resumen}

Este artículo aborda dos experiencias de visualización, resultado del encuentro y colaboración entre artistas y científicos con el objetivo de dar respuesta a cuestiones planteadas en el proyecto I+D+i "Metamétodo: Metodologías compartidas y procesos artísticos en la sociedad del conocimiento HAR2010-18453 (subprograma ARTE)", dirigido por Alicia Vela y desarrolladas por miembros del equipo IMARTE de la Facultad de Bellas Artes de la UB. La primera propuesta titulada Your colour memory se realiza en colaboración con la Unidad y Laboratorio de Enfermedades Neuromusculares del Instituto de Investigación Hospital de Sant Pau (UAB). En ella se analiza la capacidad del razonamiento científico como verdad certera indiscutible y el laboratorio como escenario donde germina dicha argumentación. El segundo estudio, titulado La pell de la pell, mediante la visualización de cromatografías y de planchas de fotopolímero sensible, establece una analogía con las "fachadas vivas", construidas de hormigón biológico dónde los líquenes son los protagonistas. El estudio surge a partir del contacto y distintas visitas al Departamento de Botánica de la Facultad de Biología de la UB.

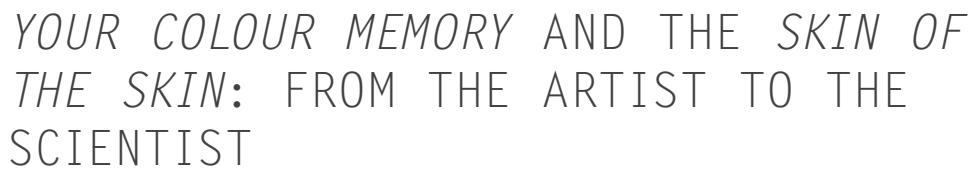

Abstract

This article explain two experiences of visualization framed within the Research and Development (I+D) project "Metamethod. Shared methodologies in artistic processes HAR 201018453 (subprogram ARTE)" developed by the artists and researchers of IMARTE Group, University of Barcelona. The first experience titled Your colour memory was made with Neuromuscular Diseases Unit and Laboratory of the Research Institute Hospital de Sant Pau (UAB). Examines the power of scientific reasoning as an unquestionable truth and the laboratory as the stage where that argument germinates. The second study, entitled The skin of the skin, starts after contact by Department of Botany, Faculty of Biology (UB). The idea of how to build living façades through biological concrete suggests an analogy from a particular visualization of the chromatographies and photopolymer plates.

Keywords: METAMETHOD; ART-SCIENCE; LIGHT; COLOR; SUSTAINABILITY

\footnotetext{
Pastó Aguilà, Cristina \& Eugènia Agustí Camí. 2016. "Your colour memory y la pel1 de 1a pel1: Del artista al científico”. AusArt 4(1): pp-pp. 197-206 DOI: 10.1387/ausart.16702
}

\section{AUSART}


La investigación artística es un espacio donde confluyen distintos lenguajes y es un campo de exploración abierto en el que dialogan diferentes disciplinas. La naturaleza de la obra de arte no tiene un único epicentro, es abierta, multidisciplinar y muestra los desplazamientos entre métodos y conceptos. Siguiendo este principio las obras que configuran "Metamétodo", comienzan con el mirar que se tornará en la observación.

"Metamétodo" reúne versatilidad y transformación. Analiza los cambios en la concepción del arte en nuestra sociedad y enfatiza el proceso de transformación de las prácticas artísticas a través de las nuevas formas de producción. Los artistas se nutren de experiencias científicas para elaborar su discurso y se interrogan sobre temas como la materia, el color, la luz, el tiempo o la visualización de datos. Es así como un artista hace visible a un científico.

\section{YOUR COLOUR MEMORY}

Your colour memory presenta un círculo transparente ideal que invade el espacio con caracteres gráficos. Estos signos de compresión y formalización geométrica traslucen la intersección discursiva de los agentes implicados en la investigación, y se hacen visibles gracias a la luz. La luz convierte en color una tinción invisible por la reactividad de un anticuerpo que reconoce una proteína. El color hace perceptible la enfermedad del tejido celular y a su vez, se sintetiza en la ilusión del círculo cromático, como las teorías que describen los colores refieren desde siglos.

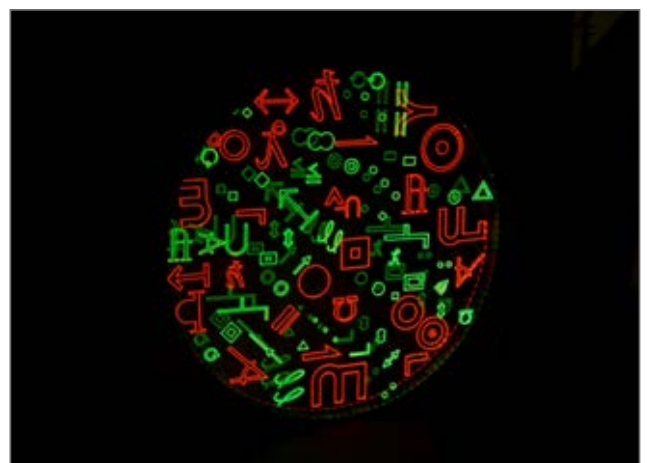

Desde nuestra perspectiva artística existe un manifiesto interés por las estructuras lingüísticas que se incrementa ante la asertividad de la teorización científica. Las atribuciones de fiabilidad y credibilidad y las conjeturas al respecto, sitúan aún más en valor el estudio de la teoría y el lugar donde se gesta, el laboratorio. Your colour memory actúa en cuatro planos: el color, la exploración de sus teo-

Fig. 1: Your colour memory (2013), Eugènia Agustí y Antònia Vilà. Dispositivo circular lumínico de metacrilato perfilado en hierro. Dimensiones: $137 \mathrm{~cm}$ de diámetro. 
rías, su examen desde la percepción científica y artística, y el intercambio desde ambos ámbitos.

Dos años de entrevistas con Eduard Gallardo y Ricardo Rojas de la Unidad y Laboratorio de Enfermedades Neuromusculares del Instituto de Investigación Hospital de Sant Pau (UAB) generan este proyecto. La esencia del encuentro adquiere un interés semiológico en la reciprocidad, en la descripción de las fases de los procesos, y en la sensibilidad al hacer un diagnóstico y su transmisión: bench to bedside ${ }^{2}$, en jerga científica. Así como los artistas se forman sobre su metodología, los científicos reciben la lectura de J. Berger Tiziano. Ninfa y pastor. Se les ofrece este texto por su desarrollo epistolar entre autores afines a la creación visual, y porque revela sus distintos saberes y experiencias sobre la pintura de Tiziano. Seleccionar este formato adquirió sentido por su estructura ilusoria y expectante, equiparable a la que mantuvieron en siglos precedentes las teorías sobre el color sostenidas por Newton, Goethe, Schopenhauer, Chevreul y Wittgenstein. Los prismas y la refracción; el surgimiento de los colores y el espectro; el ojo y la visión; la influencia de los colores en el ánimo, y la amplitud de variables sobre los presupuestos del lenguaje aplicado en sus enunciados. Todas secundan sus discursos, plasman su conocimiento sobre la luz y los colores, y enjuician su faceta fenoménica. El artista y el observador dialogan sobre el mismo mundo, y así, artista y científico comienzan a observarse.

La actividad en el laboratorio prosigue en torno a un sujeto artístico e inmunohistoquímicas de tejidos musculares cultivadas con anticuerpos, y paulatinamente, se estiman las complicidades sobre el tratamiento del color. Ello conlleva instruirse sobre fluorocromos que reaccionan a ciertas emisiones de longitudes de onda. El tejido muscular analizado y su análogo inscrito en la lente circular, comprende la secuencia de los metalenguajes que se suceden producto de los diferentes escenarios y teorías sobre la luz y el color. Durante el transcurso de la investigación se metamorfosean en los patrones codificados de las combinatorias de los factores involucrados en el estudio, y se convierten en monogramas de los referentes cromáticos, de los científicos de Sant Pau y de los artistas partícipes. Son las partículas que interactúan y emergen suspendidas en este espacio delimitado, tal y como lo hace la longitud de onda cuando dibuja la patología del tejido celular en forma verde o roja.

Al confrontar esta alegoría del examen microscópico del anticuerpo y la proteína se encadenan una serie de cuestiones: ¿Para qué son útiles las teorías del color? ¿Qué suscitan en los artistas? ¿Y en los científicos? En primera 


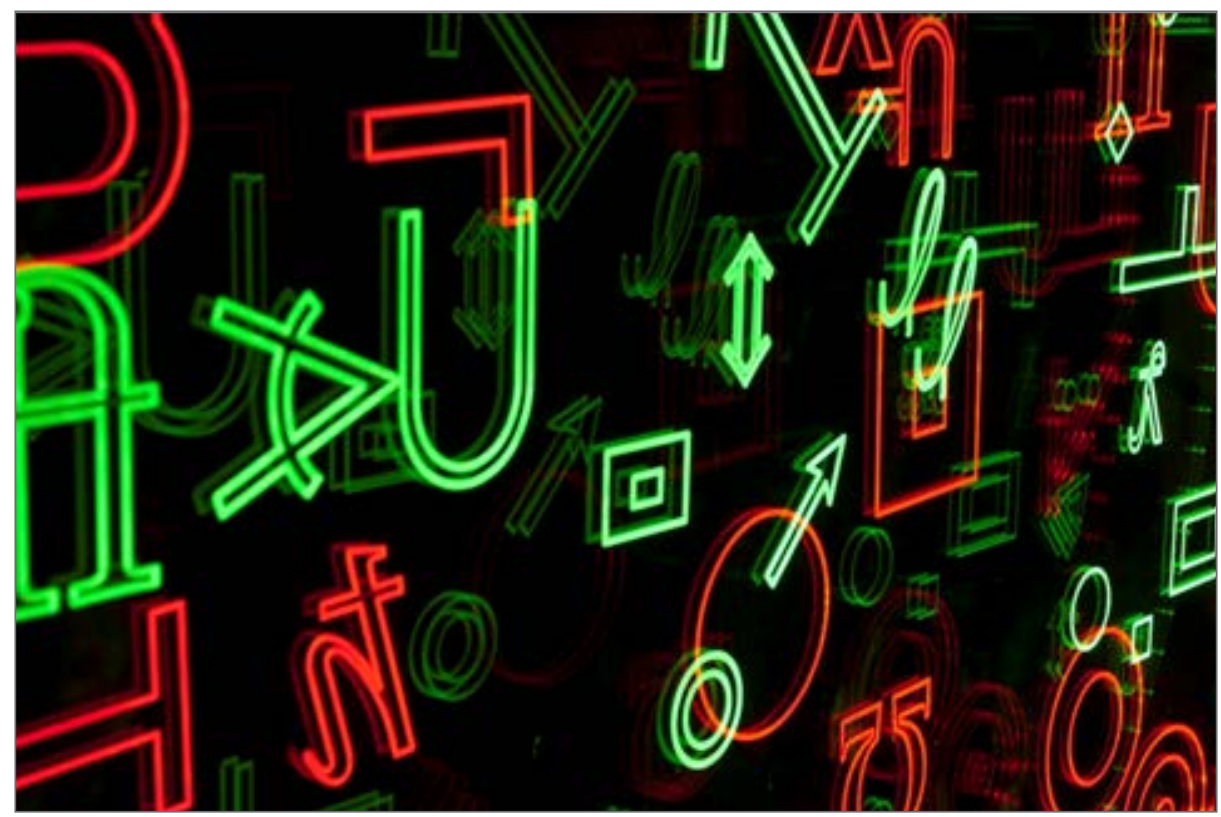

Fig. 2: Detalle de Your colour memory (2013), Eugènia Agustí y Antònia Vilà.

instancia, conforman un contexto gráfico reflexivo que nutre al artista. En segunda, afectan y estimulan su imaginación. Retomando la posición como observadores externos que contactan con el laboratorio para analizar el objeto científico, surgen interrogantes que implican al sujeto y al lugar, y por ende, a la interacción entre artistas y científicos: ¿Qué significa entrar en un laboratorio? ¿Qué relaciones genera el diálogo sobre arte y ciencia? Emplazados en el laboratorio pensamos en cómo es este escenario, y es ineludible recurrir a los textos de Latour (1983) sobre la vida en los laboratorios científicos, así como su guiño a Parménides. Latour define el laboratorio como receptáculo de observación de la realidad y lugar de transferencia de vectores: ensayo, transferencia, metáfora y transcripción. Estos se transcriben en el texto científico, así como el laboratorio es el escenario idiosincrásico en el que un científico se define como tal. Las observaciones que motivan estos estudios construyen un entramado entorno al laboratorio, en un examen de agentes que genera un razonamiento entre disciplinas que se extiende tanto a la innovación científica como a la sociedad. Ante la mitificación de las dinámicas y metodologías del laboratorio, que hacen aprehenderlo como lugar especial, Latour se centra en la idea de "construcción del laboratorio". Esta construcción deviene una revisión del lugar como "un lugar generador" de relaciones, necesariamente dialéctico desde el momento que se posiciona en el medio social. 
El modo de articular ambos lenguajes, el científico y el artístico, se establece como cadena de transmisión de sus argumentos, y la premisa de esta investigación es constatar cómo del primero al último son puestos en tela de juicio a lo largo de decenios. El estudio llevado a cabo en Your colour memory trata de hacer emerger el ojo que ve, así como lo que ve: "el laboratorio es un objeto con muchas tramas, es un gran y eficiente transformador de fuerzas" (Latour 1983). A la luz del lenguaje, lo observado en el laboratorio puede describirse con palabras, pero precisamente esa equivalencia o concordancia verbal se resiste a ser un instrumento de lo empírico. Your colour memory se sirve de sus recursos para expresar cuán complejo y cuán dinámico resulta dicho lenguaje al ser transformado.

\section{LA PELL DE LA PELL}

En La Pell de la pell. Una aproximación al estudio de los líquenes, las cromatografías usadas para clasificar líquenes y las planchas de fotopolímero sensibles a la luz, evocan la formación de un muro análogo a las llamadas "fachadas vivas" construidas con hormigón biológico donde tienen un papel fundamental los líquenes. A raíz de los encuentros con el Dr. Antonio Gómez Bolea del departamento de Botánica de la Facultad de Biología de la Universidad de Barcelona, se va gestando nuestra propuesta. Este experto en bioindicadores liquénicos participa en la investigación sobre hormigón biológico concebido para edificar fachadas con líquenes musgos y otros organismos, patentado por el Grupo de Tecnologías de Estructuras de la Universidad Politécnica de Cataluña.

El análisis del comportamiento y estructura de los líquenes, las cromatografías y las planchas de fotopolímero, sus usos y transformación, confluyen en este estudio. Destacamos que los líquenes son organismos procedentes de la asociación simbiótica entre un hongo y una alga. La protección frente a la desecación y la radiación solar que aporta el hongo y la capacidad de fotosíntesis del alga, confieren al líquen características únicas dentro de los seres vivos. El hongo, por su parte, suministra humedad al alga y un sustrato adecuado para vivir aportándole los minerales y el dióxido de carbono necesarios para fotosintetizarse. La luz es un agente decisivo para el líquen siendo indispensable para asegurar su crecimiento. Reconocido como un organismo fotófito, nece- 
sita gran cantidad de luz para compensar por vía de la fotosíntesis las pérdidas ocasionadas por la respiración.

Durante una serie de entrevistas se observan y deducen las características básicas de estos organismos cuya extraordinaria variedad de formas se revela gracias al microscopio. En las salidas de campo se localizan las especies y se toman muestras para analizar en el laboratorio con lupas y microscopios de gran precisión. Se aprende también sobre la importancia de los reactivos para reconocer los elementos químicos y distinguir las especies y sus componentes. Se descubren materiales desconocidos así como un nuevo lenguaje. Asimismo se advierte la importancia del uso de las cromatografías para reconocer las sustancias químicas que conforman cada tipo de líquen. La cromatografía es un método físico de separación de elementos. En nuestro caso, se configura por un cristal que lleva adherida una capa blanca de silicio donde se depositan muestras disueltas de líquen, y gracias a un reactivo, las sustancias de las muestras se separan y se desplazan a distintas velocidades originando una gama de colores característicos sobre la capa blanca visibles a la luz diurna (amarillos, ocres, marrones) o bajo la luz ultravioleta (azules, verdes fosforescentes). Mencionar también que en las cromatografías se da una fase móvil, en la cual un fluido arrastra la muestra de la fase estacionaria. Los componentes de la mezcla interaccionan entre sí de distinta manera y atraviesan la capa a distintas velocidades separándose.

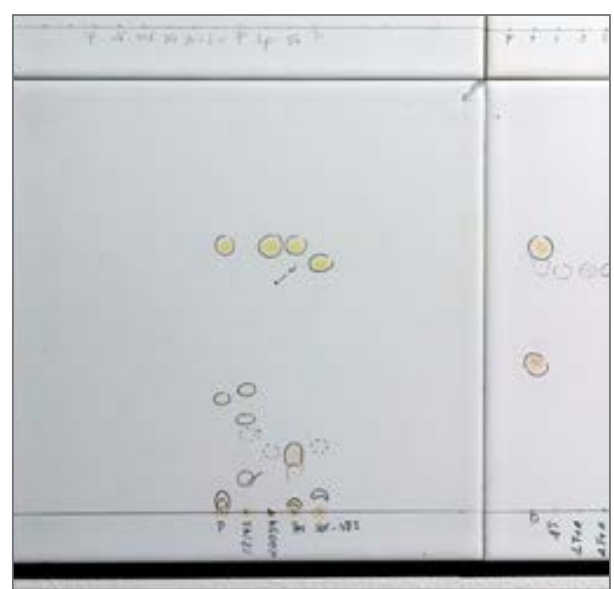

Fig. 3: Detalle de La pell de la pell (2014), Cristina Pastó. Cromatografías, $20 \times 20 \mathrm{~cm}$.
La obra presentada rememora el hormigón biológico que, sin duda, aporta una innovadora manera de hacer arquitectura. Las piezas de una edificación creadas con este material constituyen una epidermis natural, que además de funcionar como una envolvente o barrera de protección, reaccionan y varían su aspecto según su ubicación geográfica y cambios de clima producidos a lo largo del año. Las cromatografías dispuestas una tras otra, construyen una pared blanca contigua a otra pared irregular constituida por placas de polímero fotosensibles donde figuran impresas las imágenes de unos líquenes. Estas hacen alusión a un tercer muro situado físicamente en otro lugar: la fachada de hormigón biológico del 
Centro Cultural Aeronáutico del Prat del Llobregat, estructura viva que permite el crecimiento de ciertas famílias de microalgas, hongos, líquenes y musgos.

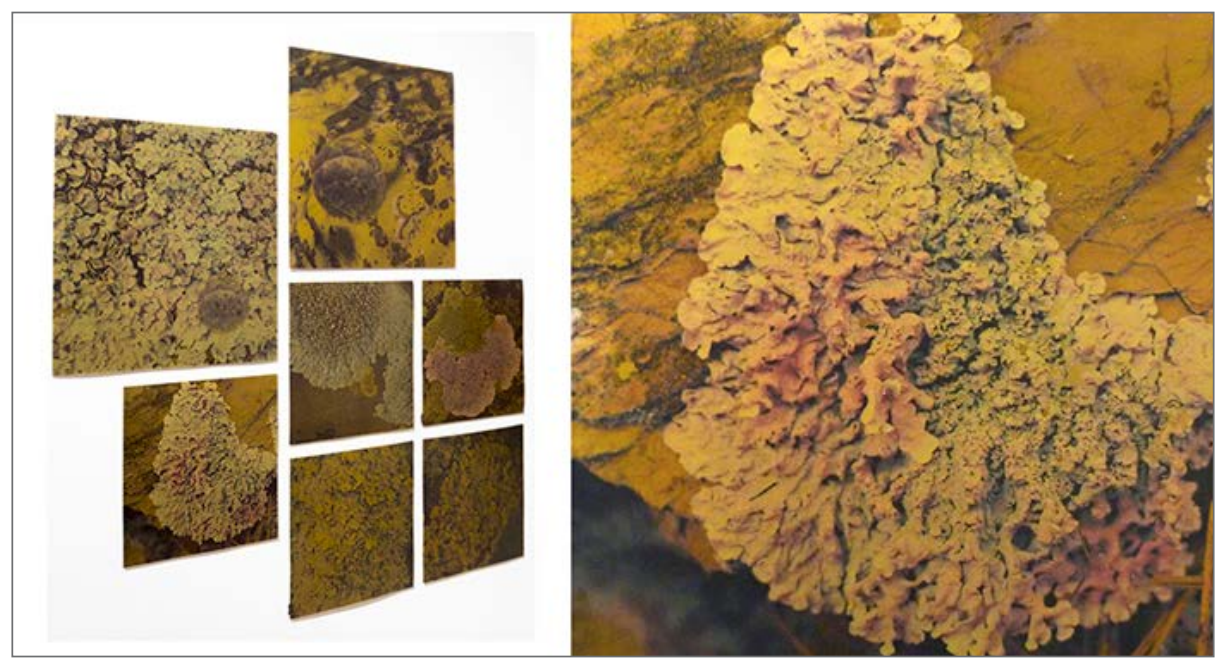

Fig. 4: Detalles de La pell de la pell (2014), Cristina Pastó. Planchas de fotopolímero de dimensiones variables.

La belleza de las placas de fotopolímero empuja al artista a buscarles nuevos usos y sentidos. Se convierten en un soporte singular de la impresión digital de alta resolución, en el cual se extienden y crecen los amarillos y naranjas de la Xanthoria parietina ${ }^{4}$. Este proyecto transita desde una pared regular de cromatografías colindante a un muro irregular de placas de polímero hacia la fachada inteligente: ese muro cambiante que deja de ser utópico donde crecen los líquenes y actuará como un gran pulmón luminoso.

Como resultado en La pell de la pell dialogan la visión fotográfica descriptiva junto al método de clasificación del científico: la cromatografía, misteriosa y bella en la cual velocidad y lentitud se complementan. La velocidad con la que corren las sustancias en las cromatografías es inversamente proporcional a la lentitud de crecimiento natural de los líquenes en su hábitat. Resulta paradigmático el pensamiento de Margulis ${ }^{5}$ con su concepto de simbiogénesis, según el cual el principal mecanismo evolutivo de innovación está en la coexistencia de dos o más especies y donde el proceso simbiótico y la cooperación son necesarios e indispensables para el desarrollo. 


\section{CONCLUSIÓN}

Your colour memory y La pell de la pell son obras que ejemplifican intercambios colaborativos en los que luz, color, forma o ambiente son sujetos determinantes y sirven de indicadores tanto para artistas como para científicos. Dar visibilidad al trabajo realizado en el laboratorio desde la mirada investigadora del artista, observando cómo concuerdan ciertas fases al tiempo que otras difieren, son objetivos logrados en aras de facilitar la transferencia de conocimiento y estimular el debate bajo otras perspectivas. Las formas de trabajo características de las dos disciplinas se ponen de relieve planteando un paradigma de fusión entre ambas. Desde la observación del mundo a la revisión de los fenómenos naturales, concluyendo en la invención de nuevos conceptos.

Your colour memory comienza con un ejemplo de investigación aplicada en que los nuevos modos de diagnosis médica también establecen nuevos modos de relación entre médico y paciente. La transferencia de ese discurso se apoya en la lectura de la imagen. Una imagen codificada que necesita de palabras para ser desentrañada. Equivalencia, traducción, interpretación o incluso traslación forman parte de la espiral del lenguaje también en el laboratorio. La visualización de la información de imágenes microscópicas de biopsias musculares en medicina es llevada al terreno metalingüístico al utilizar las tipografías como visualización alternativa. Esta instalación parte de la idea de «investigación-para», pero al mismo tiempo se organiza como «investigación-en», como práctica artística.

La pell de la pell se comporta como el portaobjetos que magnifica la conciencia sobre la sustentabilidad en la ciudad y su entorno medio ambiental, cuestionado de manera perenne. Esta producción se ha servido de medios y materiales simples y directos, que de manera progresiva se ha transformado en más complejos al ubicarse en el muro que actúa como espejo para la reflexión y la crítica.

Podemos sentir como ciencia y tecnología, en una estrecha alianza simbiótica impregnan todas y cada una de las dimensiones de nuestra actualidad, y en concreto la de la experiencia artística. Como integrantes de "IMARTE" abordamos este itinerario conscientes que los procesos de investigación que nos atañen se configuran mediante múltiples capas de conocimiento, se proveen de otros lenguajes, se nutren de ellos para procesarlos, y en última instancia traducen así su vivencia en objetos de saber. 
Las conclusiones de estas experiencias se mostraron en formato expositivo en el Centre Cultural El Carme Badalona (Barcelona) en 2014 bajo el título "Metamètode. Diàlegs entre art i ciència". Con esta ocasión se presentó la publicación Metamétodo. Metodologías compartidas en procesos artísticos que detalla estos proyectos y recoge los ensayos afines con el propósito de entender mediante la obra de arte en qué consiste hacer investigación artística. Desde el mes de marzo de 2016 estos proyectos pueden verse en una nueva exposición Metamètode. Diàlegs entre art i ciència a ESPAId'ARTS del Museu de la Ciència i de la Tècnica de Catalunya, de Terrassa (Barcelona).

\section{Referencias}

Bal, Mieke. 2009. Conceptos viajeros en las humanidades: Una guía de viaje. Traducción, Yaiza Hernández Velázquez. Murcia: Cendeac

Berger, Katya \& John Berger. (1996) 1999. Tiziano. Ninfa y pastor. Traducción, Pilar Vázquez Álvarez. Madrid: Árdora

Hediger, Irène \& Josep Perelló Palou, eds. 2010. Pensar Arte, actuar Ciencia: Artists-in-labs. Barcelona: Actar Arts Santa Mónica

Latour, Bruno. 1983. "Give me a Laboratory and I will raise the world". En Science Observed: Perspectives on the social study of Science, K. Knorr-Cetina \& M. Mullay, eds. London: Sage, $141-70$

- (1983) 2015. "Dadme un laboratorio y levantaré el mundo". Versión castellana de Marta I. González García. OEl-Programación-CTS+I-Sala de Lectura. Última modificación 14 de febrero http://www.oei.es/salactsi/latour.htm

Latour, Bruno \& Steve Woolgar. 1979. Laboratory life: The social construction of scientist facts. London: Sage

- (1979) 1995. La vida en el laboratorio: La construcción de los hechos científicos. Versión castellana de Eulalia Pérez Sedeño. Madrid: Alianza

Niedderer, Kristina \& Linden Reilly. 2010. "Research practice in Art and Design: Experimental knowledge and organised inquiry". Journal of Research Practice 6(2)

Vela Cisneros, Alicia, dir. 2014. Metamétodo: Metodologías compartidas en procesos artísticos [Meta-method: Shared methodologies in artistic processes]. Eugènia Agustí, Eloi Puig, coord. Barcelona: Comanegra

Wilson, Edward Osborne. 2014. Cartas a un joven científico. Traducción de Joan Domènec Ros. Barcelona: Debate

Zajonc, Arthur G. 1996. Atrapando la luz: Historia de la luz y de la mente. Traducción Carlos Gardini. Santiago de Chile: Andrés Bello 


\section{Notas}

1 "Metamétodo: Metodologías compartidas y procesos artísticos en la sociedad del conocimiento HAR2010-18453 (subprograma ARTE)". Plan Nacional de I+D+i 2008-2011. Subprograma de proyectos de investigación Fundamental no orientada. Subvencionado por el Ministerio de Economía y Competitividad y la Unión Europea.

${ }^{2}$ bench to bedside: de la poyata (mesa del laboratorio) a la cabecera de la cama.

${ }^{3}$ Xanthoria parietina. Líquen crustáceo, de color amarillo anaranjado a rojo, formado por lóbulos de tamaño desigual, entre 1 y $5 \mathrm{~mm}$ de ancho, cubiertos de apotecios naranjas. Talo de forma irregular, de $10 \mathrm{~cm}$ de diámetro o más. www. asturnatura.com. (consultado en 12.06.2015)

${ }^{4}$ Lynn Margulis (1938-2011). Bióloga norteamericana que destacó por una visión panorámica de la ciencia y participó en proyectos donde enlazaba la microbiología con otros campos. Convencida que el comportamiento del planeta era el de un macrorganismo complejo donde el clima, la vida y la geología actuaban de forma conjunta y se autoregulaban tendiendo al equilibrio. Concebía la evolución interpretando que los organismos que llegaban más lejos no eran los que luchaban contra los otros, sino los que cooperaban con un fin común.

${ }^{5}$ IMARTE, Equipo que desde el año 1999 viene desarrollando proyectos de investigación abordando en una primera fase los cambios que las tecnologías digitales originaban en la producción artística, recogidas en la publicación: Impresión Expandida / Expanded Print, 2010. Aquí presentamos una segunda fase que definimos como Metamétodo o metodologías compartidas y que parten de los proyectos de investigación I+D+i (HAR2010-18453) y (HAR2012-39378-C03-01-02), financiados por el Ministerio de Economía y Competividad, con fondos Feder, Universidad de Barcelona, Facultad de Bellas Artes. http//www.ub.edu/ imarte 